\title{
FINITE ELEMENT ANALYSIS ON TEMPERATURE DISTRIBUTION IN TURNING PROCESS USING DEFORM-3D
}

\author{
Yash R. Bhoyar' ${ }^{1}$, Asst. Prof. P. D. Kamble ${ }^{2}$ \\ ${ }^{1}$ M. Tech 2nd year, ${ }^{2}$ Assistant Professor, Department of Mechanical, Yeshwantrao Chavan College of Engineering, \\ Hingna Road, Nagpur, Maharashtra, India, \\ bhoyar.yash@gmail.com,pdk121180@yahoo.com
}

\begin{abstract}
The aim of this study is to create a finite element analysis simulation model in order to obtain solutions of the cutting forces, specific cutting energy and adequate temperatures occurring at different points through the chip/tool contact region and the coating/substrate boundary for a range of cutting tool materials and defined cutting conditions. Interfacial temperature in machining plays a major role in tool wear and can also result in modifications to the properties of the work piece and tool materials. As there is a general move towards dry machining, for environmental reasons, it is increasingly important to understand how machining temperature are affected by the process variables involved (cutting speed, feed rate, tool geometry, etc.) and by other factors such as tool wear.
\end{abstract}

Index Terms: Turning Process, FEA, Analytical Modeling etc...

\section{INTRODUCTION}

The turning process is used widely in industry and has countless applications. Traditionally, the process has been used to reduce the diameter of cylindrical work piece, or to change a work piece of non-circular cross-section. This is done by rotating the work piece about this of the machine's spindle and removing the work piece material with the cutting tool which is fed in the perpendicular direction. For the past fifty years metal cutting researchers have developed many modeling techniques including analytical techniques, slip-line solutions, empirical approaches and finite element techniques. In recent years, the finite element analysis has particularly become the main tool for simulating metal cutting processes. Finite element analysis are widely used for calculating the stress, strain, strain-rate and temperature distributions in the primary, secondary and tertiary sub-cutting zones. In consequence, temperatures in the tool, chip and work piece, as well as cutting forces, plastic deformation (shear angles and chip thickness), chip formation and possibly its breaking can be determined faster than using costly and time consuming experiments. In this work, FEA based models are developed that are able to predict the effect of various process variables on the performance measures of interests such as cutting forces, tool breakages, and surface accuracy.

\subsection{The Turning Process}

Turning is a very important machining process in which a single-point cutting tool removes material from the surface of a rotating cylindrical work piece. The cutting tool is feed linearly in a direction parallel to the axis of rotation. Turning is carried out on a lathe that provides the power to turn the work piece at a given rotational speed and to feed the cutting tool at a specified rate and depth of cut. Therefore, Five cutting parameters, i.e. cutting speed, feed rate, and depth of cut, tool geometry (nose radius) and cutting condition need to be determined in a turning operation for optimize parameter.

\subsection{Finite Element Analysis of Turning Process}

Finite element analysis is a most useful and accurate approach for the determination of field variables that is made possible by advancements in computational and processing power of computers and thus it is almost used for all the computer aided design methodologies in recent years. Applications range from deformation and stress analysis to field analysis of heat flux, fluid flow, magnetic flux, seepage and other flow problem. In this method of analysis, a complex region defining a continuum is discredited into simple geometric shapes called finite elements. The Present work is also based on the application of finite element for thermal analysis of single point cutting tool for turning operation. Once the model developed for determination of temperature field for single point cutting tool, it can also be implemented for other multipoint processes like drilling, milling or grinding also.

Modeling 3D cutting process using finite element techniques is an area of ongoing research activity due to significant cost savings and offers insights into the process which are not easily measured in experiments,. In particular heat transfer and the modeling of cutting process requires careful consideration in any modeling activity. This paper presents approaches for modeling the turning process for EN-24 type of steel. In this study, a Finite Element Analysis software Deform 3D is used to study the effects of cutting speed, feed rate, and type of 
alloy steel in temperature behavior. The work-piece is modeled as Elastic-plastic material to take thermal, elastic, plastic effect. Work-piece is represented by a liner model with different length for each condition.

The short material length was chosen to save computational time without compromising the model integrity as heat generation in machining is confined in small areas around the cutting zone. The work-piece shape is constructed by the DEFORM machining module, and includes geometry created by a previous tool pass, including appropriate depth of cut and nose radius details. An unstructured tetrahedral finite element mesh was generated using DEFORM's automatic mesh generation system. Re-meshing parameters, including minimum element size, and parameters for adaptive mesh definition are set within the system. For these Procedures of the simulations, a minimum element size of 0.25 of the fed rate was specified. The total number of elements was 100000 to 130000 depending upon work-piece size. The Lagrangian calculation embeds a computational mesh in the material domain and solves for the position of the mesh at discrete points in time. An incremental Lagrangian formulation with an implicit integration method designed for large deformation simulations is used to simulate the cutting process. The solver used was the sparse matrix with a direct integration method, because the conjugate-gradient offers an improved computational speed but less stability in convergence. [10] The FEA simulation of turning process with the help of DEFORM 3D is show in Figure.

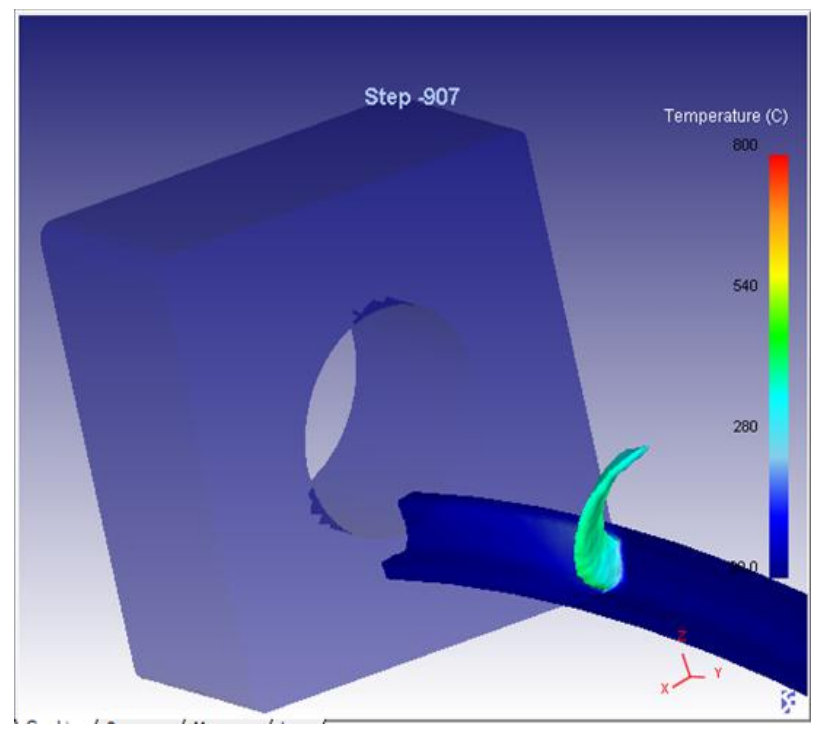

Fig.-1: Initial temperature distribution during chip formation

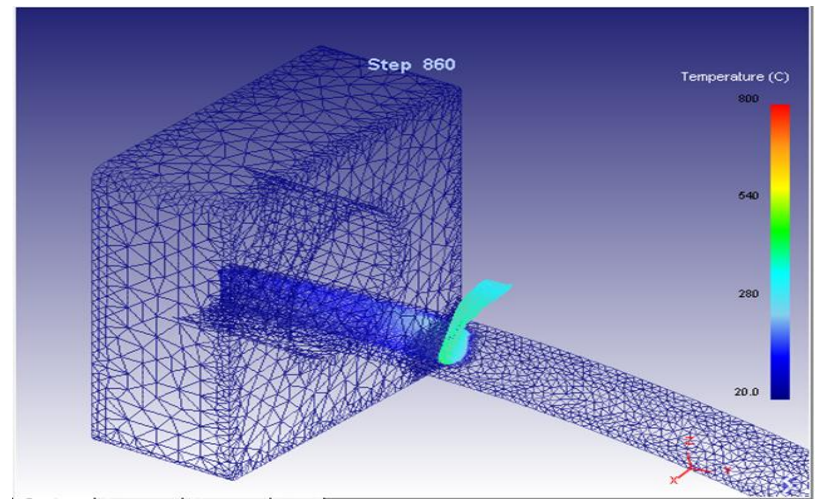

Fig.-2: Initial mesh and tool indentation

\subsection{Temperature Measurement}

The cutting speeds used in experimentation made response times of the temperature measurement devices an important criterion. The temperature measurement devices also had to be robust and have a wide measurement range. The Pyrometers is technical advances have made it possible today to measure not only high temperatures but also temperatures far below freezing point from a distance and without making contact with the object to be measured.

Pyrometer Types:

- Spectral band pyrometers

- Total band pyrometers

- Ratio Pyrometers (2 color pyrometers)

- Disappearing filament optical pyrometer (portable)

- Infrared pyrometer

- Laser pyrometer

We use Optical Infrared Pyrometer, which work on very sophisticated mechanism. This thermal device detect temperature of an object by reckoning the emitted, reflected and transmitted energy by means of optical sensors \& detectors and show temperature reading on display panel. The temperature Range is $0^{\circ}$ to $520^{\circ} \mathrm{C}$ as shown in figure.

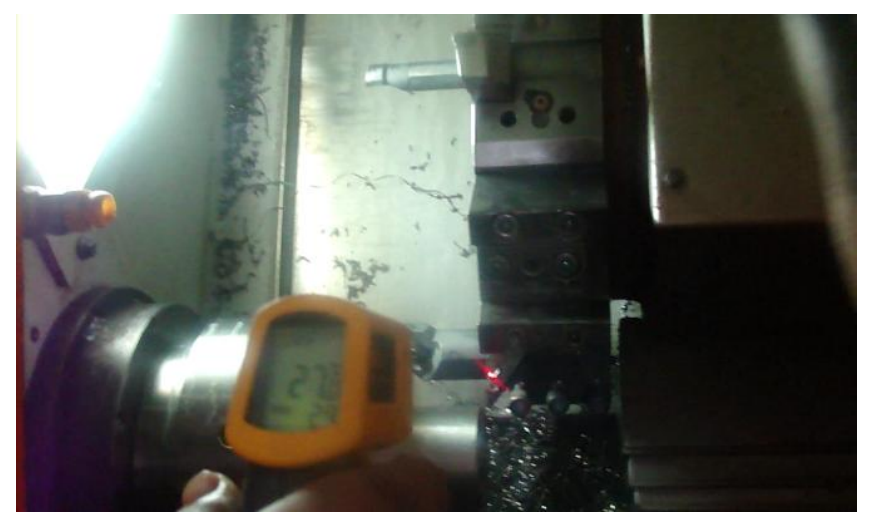

Fig.-3: Optical Infrared Pyrometer 


\section{LITERATURE REVIEW}

A Review of literature describes to study of the Temperature distribution of turning process with the help of finite element analysis.

Typical approaches for numerical modeling of metal cutting processes is Lagrangian and Eulerian techniques, as well as a combination of both called an arbitrary Lagrangian-Eulerian formulation $[2,6]$. It should be noticed that all these methods are mathematically equivalent. The major feature of Lagrangian formulation used in this study is that the mesh is attached to the work piece.

Also, the finite element analysis were performed by JohnsonCook's constitutive equation with three different sets of material constants (found by the application of several methods) is implemented in the FE model to study the behavior of Ti6Al4V alloy during the machining process in conventional and high speed regimes [4]. Demand for higher productivity and good quality for machining parts has encourage many researchers to study the effects of machining parameters using FEM simulation using either two or three dimensions version [5].

Asmaa A. kawi [10], Finite element method is a successful technique to perform analysis to estimate cutting temperatures, a possibility of developing temperature forms adequately representing metal cutting temperature as a Polynomial models of third, fourth and fifth degree with time that give steady state temperature and for the four alloys steel used and different operation conditions. All alloys have a sever increasing temperature with increasing feed rate, while it looks less sharp with increasing cutting speed .Also the ratio of the number of nodes have maximum temperature for any operating conditions and any alloy used with respect to the total number of nodes is less than $1 \%$.

Mofid Mahdi, Liangchi Zhang [11], this study considered the chip breaking and developed a 2D cutting force model with the finite element method. The variation of the cutting force was investigated carefully against both the cutting condition and the anisotropy of the material with the following development: (a) a constitutive model of a homogeneous anisotropic elastic material under plane deformation; (b) a failure model of the work material based on the Tsai Hill criterion; (c) a contact model of the mechanisms of the cutting process. A comparison with experimental measurements showed that the constitutive model leads to a reasonable prediction.

Tugrul Ozel, Taylan Altan [13], It shows a methodology to determine simultaneously (a) the flow stress at high deformation rates and temperatures that are encountered in the cutting zone, and (b) the friction at the chip-tool interface. This information is necessary to simulate high-speed machining using FEA based programs. A flow stress model based on process dependent parameters such as strain, strainrate and temperature was used together with a friction model based on shear flow stress of the workpiece at the chip-tool interface. High-speed cutting experiments and process simulations were utilized to determine the unknown parameters in flow stress and friction models.

J.C.Outeiro,J.C.Pina, R.M'Saoubi, F.Pusavec,I.S. Jawahir [14], This study presents the influence of cutting process parameters on machining performance and surface integrity generated during dry turning of Inconel 718 and austenitic stainless steel AISI 316L with coated and uncoated carbide tools. A three-dimensional Finite Element Model was also developed and the predicted results were compared with those measured.

\section{ANALYTICAL MODELING}

\subsection{Heat Balance}

In order to solve the problem with finite element method (FEM) using Deform-3D, analytical solution of the problem was reviewed by choosing each element as a control volume. From the first law of thermodynamics, the rate of difference between thermal and mechanical energy entering and exiting the control volume summing to the rate of heat generation is equal to the rate f oenergy stored within the control volume. This principal can be written as

$$
\mathrm{E}_{\text {in }}-\mathrm{E}_{\text {out }}+\mathrm{E}_{\text {generated }}=\mathrm{E}_{\text {stored }}
$$

\subsection{Heat Conduction}

The rate of heat conduction to the control volume with the dimensions of $\mathrm{dx}, \mathrm{dy}$ and $\mathrm{dz}$ from $\mathrm{x}, \mathrm{y}$ and $\mathrm{z}$ directions, are called Qx \& , Qy \& and Qz \& , respectively. The rate of heat conduction in three directions is defined as

$$
\begin{gathered}
\overline{\mathrm{Q}}_{z}=-k \cdot A \frac{\delta T}{\delta x}=-\mathrm{k} \cdot \mathrm{dy} \cdot \mathrm{dz} \frac{\delta T}{\delta x} \\
\overline{\mathrm{Q}}_{y}=-k \cdot A \frac{\delta T}{\delta y}=-\mathrm{k} \cdot \mathrm{dx} \cdot \mathrm{dz} \frac{\delta T}{\delta y} \\
\overline{\mathrm{Q}}_{x}=-k \cdot A \frac{\delta T}{\delta z}=-\mathrm{k} \cdot \mathrm{dx} \cdot \mathrm{dy} \frac{\delta T}{\delta z}
\end{gathered}
$$

Here, $\mathrm{k}$ is the thermal conductivity and A is the area of the surface that is exposed to this heat conduction.

\subsection{Heat Convection}

Another way of heat transfer is convection from the system to the surrounding air; the heat convection rate is directed from the control volume to the surrounding air which can be written as following. 


$$
\overline{\mathrm{Q}}_{\mathrm{conv}}=\text { ha } \mathrm{A}(\mathrm{T}-\mathrm{T})
$$

Here, ha is the convection coefficient between the element and the ambient air flowing around. The temperature of ambient air is considered equal to the room temperature; therefore, room temperature is boundary condition. Some elements are not in contact with air so this term becomes zero for them.

\subsection{Heat Generation}

The amount of generated heat depends on heat generation rate per control volume unit

$$
\mathrm{E}_{\text {generated }} \square=\square \text { q.dx.dy.dz }
$$

The values of generated heat are obtained from the force and velocity factors along shear and friction dimensions, as can be seen in following.

$$
\begin{gathered}
\mathrm{Q}=\mathrm{Fs} \mathrm{Vs}=\frac{\frac{\tau h V \cos \left(\alpha_{n}\right)}{\sin \left(\alpha_{n}\right) \cos \left(\Phi_{n}-\alpha_{n}\right)}}{\mathrm{Qf}=\mathrm{Ff} . \mathrm{Vc}=} \\
\frac{\cos \left(\Phi_{n}+\beta_{n}-\alpha_{n}\right) \sin \left(\Phi_{n}-\alpha_{n}\right)}{\cos \left(\beta_{n}\right)}
\end{gathered}
$$

In these equations, $\mathrm{h}$ is the uncut chip thickness which corresponds to feed rate in turning; $\mathrm{V}$ is the cutting velocity; $\tau$ is the shear flow stress; $\alpha_{n}, \beta_{n}$ and $\phi_{n}$ are the normal rake angle, normal friction angle and normal shear angle, respectively. The values of generated heat are per unit of cut; therefore, we can achieve the real values with multiplying them by depth of cut.

\subsection{Heat Stored}

The amount of heat stored within the control volume depends on volume, density $\rho$, specific heat capacity $C$, and the rate of temperature change in the control volume.

$$
\mathrm{E}_{\text {stored }}=\rho . \mathrm{C}^{\frac{\delta T}{\delta t}} \mathrm{dx} \cdot \mathrm{dy} \cdot \mathrm{dz}
$$

It is assumed that heat flows from all surrounding objects and enters to the control volume; therefore, the heat balance equation can written as follow.

$Q x+Q y+Q z+Q \operatorname{conv}+Q d x d y d z=\rho C \frac{\delta T}{\delta t} d x d y d z$

In (8), $\mathrm{Q}$ is defined as the following term.

$$
Q=q s+q f
$$

\subsection{Discretizition Of Heat Equation By Explicit Method}

The problem must be discredited in time. The integer $\mathrm{p}$ is introduced for this purpose, where $\mathrm{t}=\mathrm{p} \square \mathrm{t}$ and the finite difference approximate to the time derivative in equation is expressed as

$$
\begin{gathered}
\frac{\delta T}{\delta x}=\frac{T_{i+1, j, k}^{p}-T_{i_{j}, k}^{p}}{\Delta t} \\
\frac{\delta T}{\delta y}=\frac{T_{j+1, i, k}^{p}-T_{i_{s}, j, k}^{p}}{\Delta_{t}} \\
\frac{\delta T}{\delta z}=\frac{T_{k+1, j, i}^{p}-T_{i_{s}, j, k}^{p}}{\Delta_{t}}
\end{gathered}
$$

The superscript $\mathrm{p}$ denotes the time dependence of $\mathrm{T}$; the time derivative is expressed in terms of temperature difference between the new $(p+1)$ and previous $(p)$ times.

In the explicit method of solution, these temperatures are evaluated at the previous (p) time. Hence, Eq. 10 is considered to be a forward difference approximation to the time derivative. The accuracy of the finite difference solution may be improved by decreasing the values of nodal dimension and $\Delta_{\mathrm{t}}$.

An undesirable aspect of the explicit method is that it is not unconditionally stable. In order to prevent the explicit method from becoming unstable, the prescribed value of Dt must be maintained below a certain limit which depends on element dimensions and other parameters of the system.

\subsection{Finite Difference Equation for Tool}

In this section, after meshing of the tool, a partial differential equation is derived for each element in this grid. Notice that in the selection of the element numbers, both accuracy and time are of great importance. In the present paper, the optimum element number with proper precision is used for temperature estimation. Differential equation for one type of the elements is as the following.

$\mathrm{kA}^{,} \frac{\delta T}{\delta z}+\mathrm{kA},, \frac{\delta T}{\delta z}+\mathrm{kA},, \frac{\delta T}{\delta z}+\mathrm{h} \mathrm{A}\left(\mathrm{T}-T_{\infty}\right)=\mathrm{CV} \rho \frac{\delta T}{\delta z}$

Here, it is assumed that element dimensions are $\mathrm{Dx}=\mathrm{Dy}=$ Dz; hence, A' , A" and A"' are equal surfaces, $A=312 / 4, A^{\prime \prime}=$ $12 / 4, \mathrm{~V}=13 / 8$; Substituting these terms in (11) in a small time interval, the following equation is obtained. 


$$
\begin{aligned}
& \left.\frac{k l^{2}}{4}+\frac{T_{i+1, j, k}^{p}-T_{i, j, k}^{p}}{\Delta_{t}}\right)+\frac{k l^{2}}{4}\left(\frac{T_{j+1, i, k}^{p}-T_{i, j, k}^{p}}{\Delta_{t}}\right) \\
& +\frac{k l^{2}}{4}\left(\frac{T_{k+1, j, i}^{p}-T_{i, j, k}^{p}}{\Delta_{t}}\right)+q \frac{3 h l^{2}}{4}=\frac{\rho C l^{3}}{8}\left(\frac{T_{k, j, i}^{p+1}-T_{i, j, k}^{p}}{\Delta_{t}}\right)
\end{aligned}
$$

By solving the nodal temperature of the new $(p+1)$ time, the following is achieved.

$T_{j, i, k}^{p+1}=\frac{2 \mathrm{kt}}{\mathrm{pCl}^{2}}\left(T_{j, i+1, k}^{p}-T_{i, j+1, k}^{p}+\right.$

$T_{j, i, k+1}^{p}$ )

$+\mathrm{q}^{\frac{6 t}{\rho C l}}+\left(1-\frac{6 k t}{\rho C t^{2}}\right)\left(T_{j, i, k}^{p}\right)$

Here, some parameters are defined.

$\mathrm{F} 0=\frac{\mathrm{kt}}{\mathrm{pCl}^{2}}$

$\mathrm{Bi}=\frac{h l}{\mathrm{k}}$

Fo is Fourier number and $\mathrm{Bi}$ is Biot number, and finally we have

$$
\begin{aligned}
& T_{j, i, k}^{p+1}=2 F_{0}\left(T_{j, i+1, k}^{p}-T_{i, j+1, k}^{p}+\right. \\
& \left.T_{j, i, k+1}^{p}+3 \frac{q l}{\mathrm{k}}\right) \\
& +\left(1-6 F_{0}-6 F_{0} \times B_{i}\right) T_{j, i, k}^{p}
\end{aligned}
$$

\section{RESULT}

The temperature signals were measured by an immobile, wholly digital and fast compact pyrometer for a calibrated temperature range of -3 to +520 degrees centigrade. The laser pilot lights direct the pyrometer to the measuring position. The size of the measurement spot was $0.3 \mathrm{~mm}$, which corresponded to a distance to the measurement surface of $0.2 \mathrm{~m}$.

By Several experiments was performed in dry condition, the maximum temperature of position A1 was exceed up to 240 0C near cutting edge was observed from the optimized parameter. Similarly the temperature was decreases as far from the maximum temperature were occur position $\mathrm{A} 1$, was observed about $1900 \mathrm{C}$ at $\mathrm{A} 2$ and $1500 \mathrm{C}$ at $\mathrm{A} 3$.

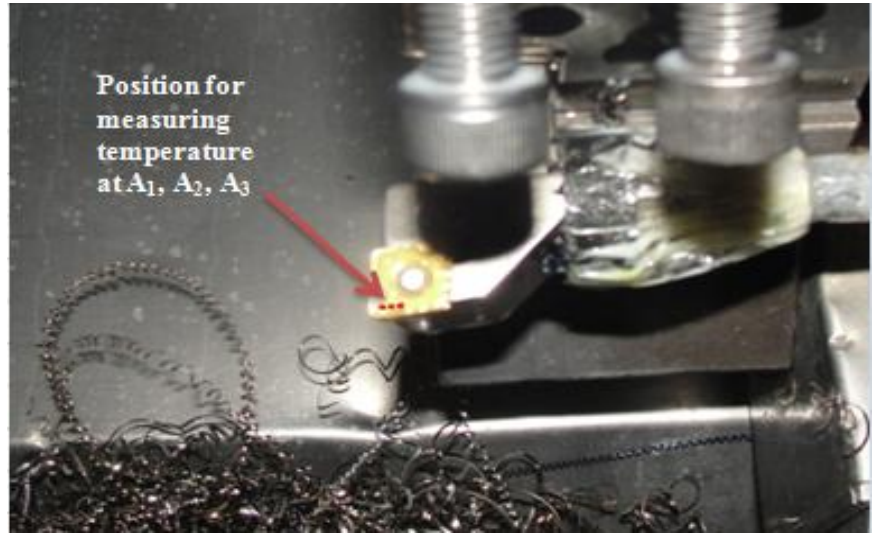

Fig.-4: Points traced for measuring temperature distribution

The Simulation of Temperature distribution in Deform-3D is perform by using Optimized Parameter as follows,

- $\quad$ Speed $-158 \mathrm{~m} / \mathrm{min}$

- Feed $-0.2 \mathrm{~mm} / \mathrm{rev}$

- $\quad$ DOC - $2 \mathrm{~mm}$

- $\quad \mathrm{NR}-1 \mathrm{~mm}$

- Condition - Dry

By solving analysis on Deform-3D we can find temperature distribution at any position using point tracking method in Deform-3D, fig.-6 shows the comparison of temperature results between experiments and simulation.

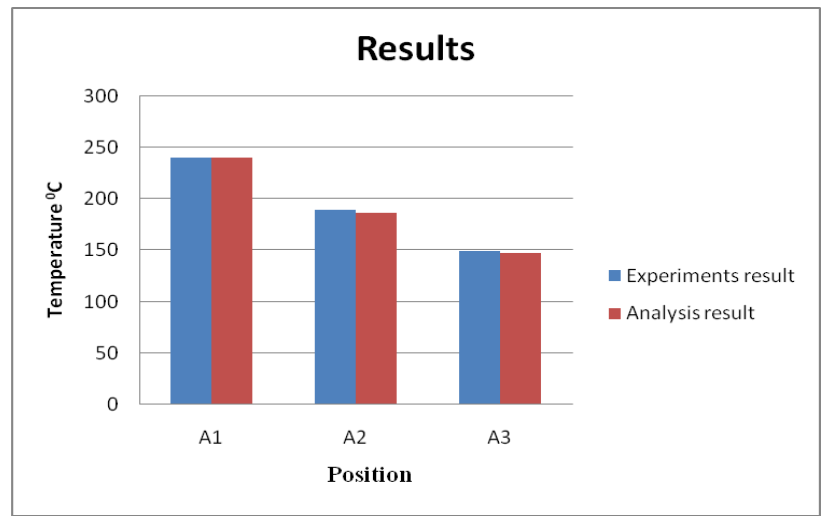

Fig.-5: results of Experiments and Simulation

\section{CONCLUSIONS}

The following conclusions could be drawn from the results of present study:

1) Finite element method using DEFORM 3D program is found to be a successful technique to perform trend analysis to estimate cutting temperatures in metal cutting with respect to various combinations of design variables (metal cutting speed, feed rate and depth of cut). 
2) In consequence, the maximum interface temperature exists in the vicinity of the cutting edge i.e. in the first part of the tool-chip contact.

3) Increased cutting speeds $(\mathrm{Vc})$ resulted in decreased cutting tool forces and machined surface temperatures.

4) Tool wear resulted in increased cutting tool forces and machined surface temperature.

5) Force has been found to be an important variable in the generation of surface temperature.

6) Thus, it is possible to increase machine utilization and decrease production cost in an automated Manufacturing environment.

7) The formation of built-up layers in metal cutting processes is very common with a variety of layers formed having different compositions and effectiveness in reducing cutting tool wear.

\section{ACKNOWLEDGMENTS}

I express my sincere gratitude to my guide, Prof. P. D. Kamble, Asst. Professor, Mechanical Department, Yeshwantrao Chavan College of Engineering for his valuable guidance, proper advice, and careful reviews of my work at all stages, and their highly appreciated instruction and constant encouragement during the course of my work on this paper.

I am highly thankful to Prof. B. D. Deshmukh, Professor and H.O.D., Mechanical Department, Yeshwantrao Chavan College of Engineering for his expert advice, technical suggestions and moral support during in this work.

\section{REFERENCES}

[1] W. Grzesik, M. Bartoszuk and P. Niesłony. Finite element modeling of temperature distribution in the cutting zone in turning processes with differently coated tools., $13^{\text {th }}$ international scientific conference. 2005.pp 1-4

[2] M. H. El-Axir . A methode of modeling residual stress distribution in turning for different material., International journal of Machine tool \& Manufacture 42 (2002) 1055-1063

[3] S. R. Carvalho, S. M. M. Lima e Silva, A. R. Machado, G. Guimaraes. Temperature determination at the chiptool interface using an inverse thermal model considering the tooland tool holder., Journal of Material Processing Technology 179 (2006)

[4] Domenico Umbrello. Finite element simulation of conventional and high speed machining of Ti6Al4V alloy., journal of materials processing technology 196 (2008) 79-87

[5] Hendri Yandra ${ }^{1}$, Jaharah A. Ghani ${ }^{2}$ \& Che Hassan Haron. Effect of Rake angle on Stress, Strain and Temperature on the Edge of carbide cutting tool in Orthogonal Cutting using FEM Simulation., ITB J. Eng. Sci., vol. 42, No.2, 2010
[6] O. Pantal_e, J.-L. Bacaria,O.Dalverny, R.Rakotomalala, S. Caperaa. 2D and 3D numerical models of metal cutting with damage effects., Comput. Methods Appl. Mech. Engrg. 193 (2004)

[7] Jaroslav Mackerle. Finite-element analysis and simulation of machining: a bibliography., Journal of Materials Processing Technology 86 (1999) 17-44

[8] W. H. Yang, Y. S. Tarng. Design optimization of cutting parameters for turning operations based on Taguchi Method. Journal of Processing Technilogy 84 (1998) 122-129

[9] Dr.S.S.Chaudhari, S.S. Khedkar, N.B. Borkar. Optimization of process parameters using Taguchi approach with minimum quantity lubrication for turning. International Journal of Engineering Research and Applications (IJERA). Vol. 1, Issue 4, pp.12681273

[10] Asmaa A. Kawi. Temperature behavioure of some alloy steels in Turning Process under different operating conditions., Al-Qadisiya Journal for Engineering Sciences, 2011 Vol. 4 No. 3

[11] Mohid Mahdi, Liangchi Zhang. A Finite element model for the orthogonal cutting of fiber-reinforced composite materials.,Journal of Material Processing Technology 113 (2001) 373-377

[12] H.S. Qi, B. Mills. Formation of a transfer layer at the tool-chip interface during machining., Wear 245 (2000) 136-147

[13] Tugrul Ozel,Taylan Altan. Determination of workpiece flow stress and friction at the chip-tool contact for high- speed cutting., International Journal of Machine Tools \&Manufacture 40 (2000) 133-152

[14] J.C.Outeiro,J.C.Pina,R.M'Saoubi,F.Pusavec,I.S. Jawahir. Analysis of residual stresses induced by dry turning of difficult-to-machine materials., CIRP Annals - Manufacturing Technology 57 (2008) 77-80 